\title{
Segmentation and quantification of retinal lesions in age-related macular degeneration using polarization- sensitive optical coherence tomography
}

\author{
Bernhard Baumann \\ Erich Götzinger \\ Michael Pircher \\ Harald Sattmann \\ Medical University of Vienna \\ Center for Medical Physics and Biomedical Engineering \\ Währinger Straße 13 \\ A-1090 Vienna, Austria
}

\author{
Christopher Schütze \\ Ferdinand Schlanitz \\ Christian Ahlers \\ Ursula Schmidt-Erfurth \\ Medical University of Vienna \\ Department of Ophthalmology \\ Währinger Gürtel 18-20 \\ A-1090 Vienna, Austria
}

\author{
Christoph K. Hitzenberger \\ Medical University of Vienna \\ Center for Medical Physics and Biomedical Engineering \\ Währinger Straße 13
}

A-1090 Vienna, Austria

\begin{abstract}
We present polarization-sensitive optical coherence tomography (PS-OCT) for quantitative assessment of retinal pathologies in age-related macular degeneration (AMD). On the basis of the polarization scrambling characteristics of the retinal pigment epithelium, novel segmentation algorithms were developed that allow one to segment pathologic features such as drusen and atrophic zones in dry AMD as well as to determine their dimensions. Results from measurements in the eyes of AMD patients prove the ability of PS-OCT for quantitative imaging based on the retinal features polarizing properties. Repeatability measurements were performed in retinas diagnosed with drusen and geographic atrophy in order to evaluate the performance of the described methods. PS-OCT appears as a promising imaging modality for three-dimensional retinal imaging and ranging with additional contrast based on the structures' tissue-inherent polarization properties. (C) 2010 Society of Photo-Optical Instrumentation Engineers. [DOI: 10.1117/1.3499420]
\end{abstract}

Keywords: optical coherence tomography; polarization sensitive devices; medical imaging; ophthalmology; algorithms; segmentation; age-related macular degeneration.

Paper 10044SSPR received Jan. 29, 2010; revised manuscript received May 22, 2010; accepted for publication Jun. 1, 2010; published online Nov. 12, 2010.

\section{Introduction}

During the last two decades, optical coherence tomography (OCT) evolved as a noncontact technique for imaging transparent and translucent samples with micrometer-scale resolution. ${ }^{1}$ OCT is still subject to permanent enhancements in order to increase imaging performance, and a wide range of applications has opened in biomedical imaging. 2,3 Mainly in ophthalmology, OCT has emerged as an indispensable tool for diagnosis of eye disease and for monitoring during therapy. ${ }^{4}$ Recent advances in terms of sensitivity and imaging speed using spectral domain (SD) OCT allow one to record three-dimensional (3-D) data sets comprising several tens of thousands of depth profiles within a few seconds. ${ }^{5-7}$

In clinical routine, apart from 2-D imaging modalities such as fundus photography, scanning laser ophthalmoscopy, fluorescence angiography, and fundus autofluorescence, OCT is used for retinal imaging in three dimensions. Since reviewing and interpretation of large 3-D data sets may be costly in terms of time and effort, several attempts have been made to ease their analysis. Anatomical characteristics and parameters such as retinal nerve fiber layer thickness or the macular volume can be determined from OCT data sets recorded in the posterior

Address all correspondence to: Bernhard Baumann or Christoph Hitzenberger, Medical University of Vienna, Center for Medical Physics and Biomedical Engineering, Währinger Straße 13, A-1090 Vienna, Austria. Tel: 43-1-427760728; Fax: 43-1-4277-9607. E-mail: bernhard.baumann@meduniwien.ac.at or christoph.hitzenberger@meduniwien.ac.at. eye segment. Retrieval of such parameters alleviates the extraction and evaluation of possible pathological changes in ocular structures. Therefore, in recent years, segmentation of features in OCT data sets has gained particular interest. ${ }^{8-15}$ Most of the approaches for segmentation of retinal layers are based on reflectivity dissimilarity of different tissue types. However, while the layered structure of the retina is well ordered in healthy eyes, it is sometimes hard to differentiate structures solely based on their reflectivity in eyes with retinal disease. Nevertheless, socalled functional extensions of OCT have been shown to provide additional contrast by being able to record not only reflectivity profiles but also depth-resolved information about flow velocity, changes in the probe light's wavelength spectrum, or the polarization properties of tissues as in Doppler OCT, spectroscopic OCT, and polarization-sensitive (PS) OCT, respectively. ${ }^{16-20}$ In the human retina, birefringent, polarization-preserving, and depolarizing structures can be distinguished by PS-OCT. ${ }^{21-25} \mathrm{Re}-$ cently, segmentation of the retinal pigment epithelium (RPE) was demonstrated by PS-OCT based on that layer's-in contrast to most of the other layers - depolarizing character. ${ }^{26}$ Using this tissue-specific contrast, our group showed the potential of PS-OCT to image the RPE in patients with age-related macular degeneration (AMD). ${ }^{27,28}$

AMD is a multifactoral disease that results in progressive vision loss in the central region of the retina (i.e., the macula). AMD is the leading cause of blindness and severe vision

1083-3668/2010/15(6)/061704/9/\$25.00 @ 2010 SPIE 


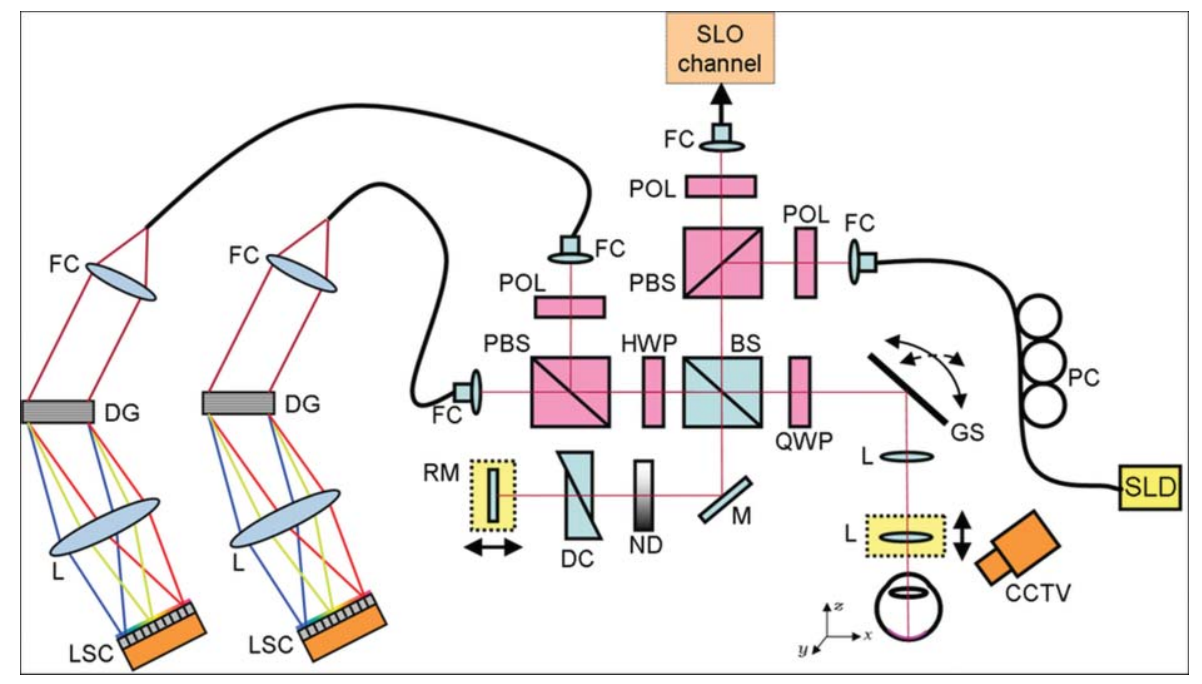

Fig. 1 Combined PS-OCT and SLO instrument for clinical imaging. Components: SLD, superluminescent diode; PC, polarization controller; FC, fiber coupler; POL, polarizer; PBS, polarizing beamsplitter; BS, nonpolarizing beamsplitter; QWP, quarter-wave-plate; GS, galvanometer scanner; $\mathrm{L}$, lens; $\mathrm{M}$, mirror; ND, variable neutral density filter; DC, dispersion compensation; RM, reference mirror; HWP, half-wave plate; DG, diffraction grating; LSC, line-scan camera; and CCTV, camera for pupil observation.

loss in the industrialized world's elderly population. ${ }^{29}$ In the early stages of the disease, metabolic endproducts accumulate in Bruch's membrane and build up a hydrophobic barrier between the posterior vascular layers and the RPE and, therewith, the retina. RPE detachments can result from these deposits, which are called drusen. ${ }^{30}$

At late stages, AMD is classified as wet (neovascular, exudative) or dry (nonexudative) AMD. The latter branch, which accounts for $80-90 \%$ of all diagnosed patients, is characterized by sharply delineated areas of severe depigmentation or apparent absence of the RPE with a minimum diameter of $175 \mu \mathrm{m}$. Wet AMD - although accounting for only $10-20 \%$ of all patientsis responsible for $80 \%$ of vision loss by AMD. ${ }^{31}$ It is characterized by RPE detachments, subretinal or sub-RPE neovascular membranes, scar tissue, subretinal hemorrhages, and related hard exudates (lipids). ${ }^{30}$

The RPE plays the key role in all types of AMD. In this paper, we demonstrate the potential of PS-OCT as a tool for not only 3-D high-resolution retinal imaging with additional contrast but also for quantitative assessment of retinal disorders in AMD. On the basis of the additional contrast provided by PS-OCT, software algorithms have been developed for quantification of clinically relevant parameters such as the area and volume of drusen and the area of atrophic zones in geographic atrophy. After a description of the PS-OCT device and the segmentation algorithms in Section 2, results from measurements in patients, including a repeatability analysis, are presented in Section 3 and discussed in Section 4.

\section{Materials and Methods}

\subsection{PS-OCT Instrument}

A spectral domain PS-OCT setup was used for imaging, a scheme of which is shown in Fig. 1. A superluminescent diode (Superlum Diodes, Inc.) centered at $839 \mathrm{~nm}$ with a FWHM bandwidth of $58 \mathrm{~nm}$ was used as a light source. The light was inserted into a Michelson interferometer in a vertically polarized state using a bulk optics polarizer. After passing a polarizing beamsplitter (PBS), the light was split into a sample and a reference beam by means of a nonpolarizing beamsplitter (BS). In the sample arm, a quarter wave plate (QWP) was used in order to provide circularly polarized light onto the eye. Further sample arm optics included an $X-Y$ galvanometer scanner and a relay of two identical achromat lenses. A probe power of $700 \mu \mathrm{W}$ on the cornea was used, which is well below the limits drawn by American National Standards Institute (ANSI) and International Electrotechnical Commission (IEC). ${ }^{32,33}$ Half of the light beam returning from the sample arm was again directed into the source arm. There, its horizontally polarized portion was detected by an avalanche photodiode (APD), in order to be able to record scanning laser ophthalmoscope (SLO) images using the same sample arm optics.

In the reference arm, the beam passed a variable neutral density filter, glass prisms for dispersion compensation, and was reflected by a mirror mounted on a motorized translation stage. Light returning from sample and reference arm, respectively, interfered at the nonpolarizing BS. Note that the QWP present in the reference arm of the original PS interferometer scheme devised by Hee et al. ${ }^{19}$ was omitted in our design in order to avoid saturation of the APD by light returning from the reference mirror. This altered layout required an additional half-wave plate (HWP) oriented at $22.5 \mathrm{deg}$ in the detection arm, which rotated the beam's polarization state by $45 \mathrm{deg}$ in front of the PBS in the detection arm, such that equal reference arm power was provided in both orthogonal polarization channels. In a software compensation step, this rotation was compensated for by multiplying the OCT signals from the two channels with the Jones matrix of a HWP oriented at $-45 \mathrm{deg}$. Now, the previously published method for computing PS-OCT signals could be applied. ${ }^{34}$

From the interferometer exit, the signals of the two orthogonal polarization channels were guided to two identical spectrometer units employing diffraction gratings with 1200 lines $/ \mathrm{mm}$ and achromat lenses $(f=200 \mathrm{~mm})$. The spectral interference 
signals were read out simultaneously at two line-scan cameras (e2v AViiVA M2 CL 2014) operating at $20 \mathrm{kHz}$. Each readout of the two cameras yielded the spectral data necessary to compute not only reflectivity but also phase retardation, axis orientation, and Stokes vectors for one depth profile.

Two operational modes were available for imaging, which were timed and controlled using a field-programmable gate array (FPGA). For alignment of the eye under investigation, SLO images and OCT B-scan images were recorded and displayed in an alternate fashion to provide an overview in three dimentions. SLO and OCT images were recorded in 165 and $20 \mathrm{~ms}$, respectively. In the real-time display, each SLO image was followed by four OCT images. Once the eye was aligned properly, 3-D data sets covering a retinal cube of $6.2 \times 6.7 \times 3.3 \mathrm{~mm}^{3}$ $(x \times y \times z)$ were recorded. One out of three sampling patterns $(64 \times 1024,128 \times 512,256 \times 256)$ could be selected. For this study, only data sets sampled $128 \times 512$ (that is, $128 \mathrm{~B}$-scans each consisting of 512 A-lines) were used.

The system was used for imaging in the eyes of patients suffering from various ocular diseases. Compliant patients diagnosed with drusen and advanced dry AMD (geographic atrophy), respectively, were selected and imaged for this study. The study protocol was approved by the ethics committee of the Medical University of Vienna and followed the tenets of the declaration of Helsinki.

\subsection{Degree of Polarization Uniformity}

Segmentation of the RPE was performed based on its depolarizing (i.e., polarization scrambling), character as reported in Ref. 26. For each B-scan, the signals recorded at the two exits of the PS-OCT instrument were processed using regular SD-OCT processing, including subtraction of an averaged spectrum, rescaling, numerical dispersion compensation, and Fourier transform. ${ }^{35}$ From the resulting signals, $I_{1,2}(z)=A_{1,2}(z)$ $\exp \left[i \Phi_{1,2}(z)\right]$, the Stokes vector $S=(I Q U V)^{\mathrm{T}}$ was computed for each image pixel. In order to gate out noisy pixels, for further processing, only pixels with intensities above a depth-dependent threshold $I_{\text {thr }}(z)=I_{\text {thr }}(z=0 \mu \mathrm{m})-z \cdot 1.7 \times 10^{-3} \mathrm{~dB} / \mu \mathrm{m}$ were considered. $I_{\text {thr }}(z=0 \mu \mathrm{m})$ was set to a value of $\sim 11 \mathrm{~dB}$ above a level equal to two standard deviations of the average noise amplitude in each A-scan. ${ }^{36}$

Each of the Stokes vector elements $Q, U$, and $V$ was normalized by $I$ and then averaged in a sliding window sized $8(x) \times 5(z)$ pixels $\left(\sim 100 \times 16 \mu \mathrm{m}^{2}\right)$. Thereof, the degree of polarization uniformity (DOPU) was calculated as

$$
\operatorname{DOPU}(z)=\sqrt{Q_{\text {mean }}^{2}(z)+U_{\text {mean }}^{2}(z)+V_{\text {mean }}^{2}(z)} .
$$

DOPU has a form similar to the degree of polarization known from conventional polarization optics. Dependent on the uniformity (or nonuniformity) of the polarization states of pixels inside of the respective evaluation window, DOPU values will exhibit values in the range from 0 to 1 . DOPU values close to 1 will be found in polarization preserving and in birefringent structures whereas polarization scrambling (depolarization) will manifest in lower DOPU values.

Now, from each DOPU B-scan image, depolarizing structures can be derived by segmenting pixels with low DOPU values, that is, pixels with DOPU values below a threshold
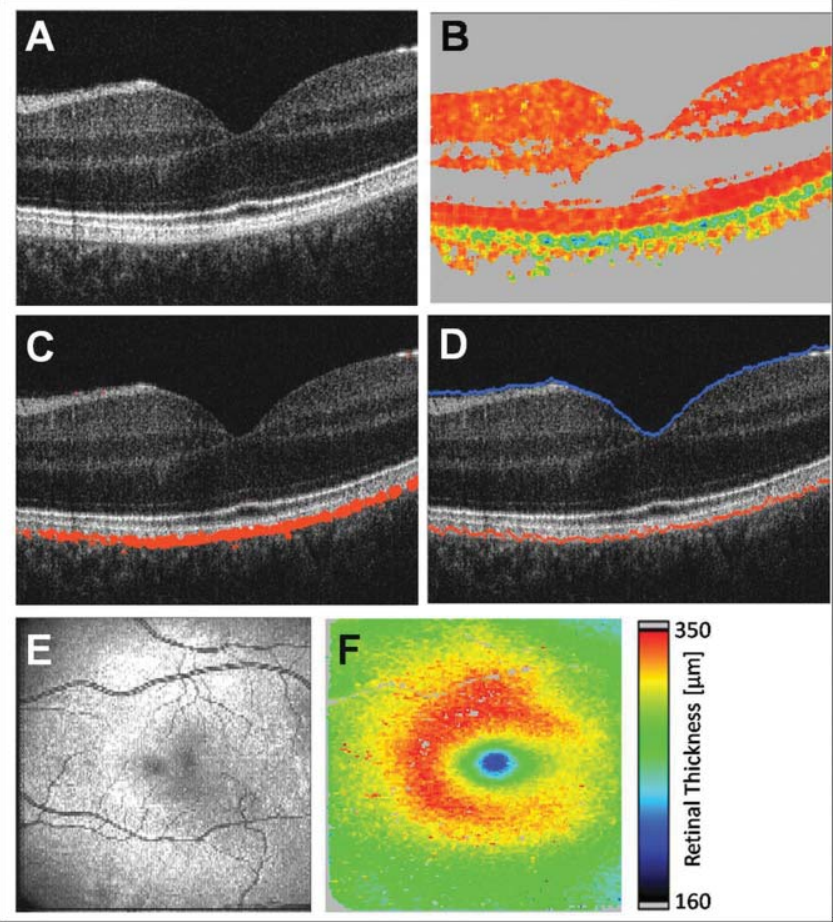

Fig. 2 PS-OCT images. (a) Reflectivity B-scan image. (b) DOPU B-scan image [color scale: DOPU $=0$ (dark blue) to DOPU $=1$ (red)]. Pixels with intensities below a certain threshold are displayed in gray. (c) Overlay of depolarizing pixels on reflectivity image in red. (d) Position of ILM and RPE overlaid on reflectivity image in blue and red, respectively. (e) En face fundus image reconstructed from 3D OCT data set. (f) Retinal thickness map computed as geometric axial distance between ILM position and RPE position.

DOPU $_{\text {thr. }}$ Segmentation results may vary, depending on the choice of DOPU $\mathrm{thr}_{\mathrm{th}}$, and the optimal DOPU $\mathrm{thr}_{\mathrm{th}}$ value can be different, depending on the respective eye and on the image quality. For the results and figures in this paper, a fixed value of 0.8 was chosen for DOPU $\mathrm{thr}_{\text {. }}$

Figure 2 shows PS-OCT B-scan images of a human retina in the fovea region of a healthy volunteer's left eye. In Fig. 2(a), the reflectivity B-scan image is displayed where healthy layers can be distinguished based on the intensity of backscattered light. The respective DOPU image is shown in Fig. 2(b). While most layers-those appearing in reddish color-do not considerably alter the sample beam's polarization state, polarization-scrambling manifests in lower DOPU values displayed in green and blue. Note that, due to the computation of DOPU by averaging in a sliding evaluation window, the spatial resolution is somewhat reduced in this image. By considering only pixels with low DOPU values, depolarizing structures can be segmented as shown in Fig. 2(c), where low-DOPU-valued pixels are overlaid in red. In Fig. 2(d), the position of the internal limiting membrane (ILM), which was found by intensity thresholding, is overlaid on the reflectivity image in blue. Furthermore, the position of the pixel with the lowest DOPU value in each Aline (i.e., the pixel exhibiting the highest depolarization), which in the healthy retina is located in the RPE, is displayed in red. A fundus image computed from the OCT data set by axial summation of the intensity for each A-line is shown in Fig. 2(e), 
and in Fig. 2(f), a retinal thickness map derived by calculating the axial distance between ILM and RPE position can be seen. An average refractive index of 1.38 was assumed in the retina.

\subsection{Segmentation of Drusen}

Drusen were segmented by first locating the actual position of the RPE by its DOPU value. This line, found by tissue-specific contrast, acts as a "backbone" for the following calculation of the position where the RPE should be in a healthy eye. Finally, the drusen are segmented and quantified by calculating the difference between the actual position and the normal "should-be" position.

For each B-scan, the starting point of the calculation is the axial position $Z_{\mathrm{RPE}}(i)$ of the minimum DOPU value in each A-line, that is, the red line in Fig. 2(d). The index $i$ indicates the index of the A-line in the respective $\mathrm{B}$-scan and $Z_{\mathrm{RPE}}(i)$ is measured as the distance from the zero delay, which is located at the upper border of all shown B-scan images, and $Z_{\mathrm{RPE}}(i)$ increases in the posterior direction. First, missing values of $Z_{\mathrm{RPE}}(i)$ are interpolated in A-scans, where no pixel is below

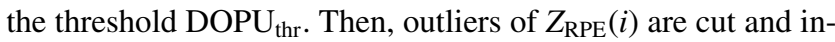
terpolated. Outliers are defined as values $Z_{\mathrm{RPE}}(i)$, which differ by $>33 \mu \mathrm{m}$ (i.e., 10 pixels) either from the mean of $Z_{\mathrm{RPE}}(i-1)$ and $Z_{\mathrm{RPE}}(i-1)$ or from the mean of $Z_{\mathrm{RPE}}(i+1)$ and $Z_{\mathrm{RPE}}(i+2)$, or from both. If $Z_{\mathrm{RPE}}(i)$ is an outlier, then $Z_{\mathrm{RPE}}(i)$ is replaced by the average of its four neighboring values $Z_{\mathrm{RPE}}(i+m)$, where $m=-2,-1,+1,+2$. The subsequent function is smoothed using a Savitzky-Golay filter (polynomial order: 3, filter length: 6).

The result of these first steps is a function $\hat{Z}_{\mathrm{RPE}}(i)$ without gaps or outliers. The following part of the computation is an iterative approximation of the normal RPE position. Every iteration of the loop comprises two steps: In the first step, the output $B_{j-1}$ of the previous loop iteration is used as an input. For the first iteration, the input $A_{0}(i)=\max _{i} Z_{\mathrm{RPE}}(i) \forall i$ is used. Now,

$$
A_{j}(i)\left\{\begin{array}{cl}
B_{j-1}(i), & B_{j-1}(i)-Z_{\mathrm{RPE}}(i) \leq 10 \Delta z_{\mathrm{pix}} \\
B_{j-1}(i)-\Delta z_{\mathrm{pix}}, & B_{j-1}(i)-Z_{\mathrm{RPE}}(i)>10 \Delta z_{\mathrm{pix}}
\end{array}\right.
$$

where $\Delta z_{\text {pix }}$ is the axial extension of one pixel $(\sim 3.2 \mu \mathrm{m}$, optical distance) and $j \in[1,175]$ is the respective iteration number of the loop. The offset $10 \Delta z_{\text {pix }}$ was chosen to compensate for flattening effect of the subsequent smoothing step.

The new function $A_{j}$ is used in the following second step to compute the output $B_{j}$ :

$$
B_{j}=\mathrm{SG}\left(A_{j}\right)
$$

where a smoothing Savitzky-Golay (SG) filter is represented with a window length of 200 using a third-order polynomial. As the loop is iterated 175 times, $B_{j}$ converges toward a smooth function resembling the normal RPE position. As a final point, $B_{175}+10 \Delta z_{\text {pix }}$ yields the final function $Z_{\mathrm{NRPE}}$. Here, the offset $10 \Delta z_{\text {pix }}$ accommodates for an axial shift of the smoothed function $B_{175}$.

Drusen can now be found by computing the difference between actual RPE position $Z_{\mathrm{RPE}}$ and normal RPE position $Z_{\mathrm{NRPE}}$ for each A-line. In the case of very large drusen or drusenoid pigment epithelium detachments in retinas appearing heavily bent in the OCT B-scan image, $Z_{\mathrm{NRPE}}$ may be displaced in anterior direction (i.e., between the actual position of Bruch's membrane and the RPE). This will not affect the calculated drusen area, but the volume may be underestimated. We observed this effect in confluent drusen with a diameter of $\sim 3 \mathrm{~mm}$. For the results presented in this study, which included patients with drusen classified as AREDS score 2 and 3, this effect was not apparent.

\subsection{Segmentation of Geographic Atrophy}

Using PS-OCT, atrophic zones in dry AMD can be detected by detecting holes in the depolarizing RPE layer.

In a straightforward manner, one could simply sum the number of depolarizing pixels [i.e., the red pixels in Fig. 2(c)] for each A-line of a 3-D PS-OCT data set. The resulting thickness map of the depolarizing layer now reveals the existence and absence of polarization scrambling tissue in an en face view.

However, depending on the subject's pigmentation, depolarization may also be apparent in the choroid. Signals from this layer usually are rather weak when the probe beam anteriorly has to penetrate the highly scattering and absorbing RPE. Contrarily, in eyes with geographic atrophy, the missing RPE usually results in higher penetration depths (sometimes down to the sclera) and in stronger OCT signals from the choroid. Dependent on the subject, choroidal tissue exhibits a more or less polarization scrambling character. In some cases, depolarizing spots with DOPU values as low as those in RPE tissue may be noticeable. If, in such eyes, the number of all depolarizing pixels is integrated over depth in order to compute a thickness map of the RPE, then polarization scrambling pixels in the choroid may add to those in the RPE. This can result in mimicking of healthy structures and masking of RPE defects, if the RPE is absent or thinned as in the case of geographic atrophy.

To prevent from integrating choroidal pixels into computing of the thickness map of the depolarizing layer, only depolarizing pixels located in a shallow band close to the photoreceptor layer and Bruch's membrane should be contributing.

For this purpose, the ILM position is used as a starting point. The initial ILM position is smoothed similar to the RPE position in the previous section by using a third-order polynomial SG filter with a window length of 200 , yielding the function

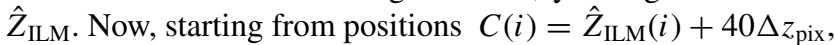
hyperintense structures are detected by seeking the first pixel with an intensity greater than the threshold computed as the average of the maximum pixel values of each A-line minus $7 \mathrm{~dB}$. Typically, the positions in the photoreceptor/RPE complex or Bruch's membrane are detected. Subsequently, discontinuities are interpolated and, similar to the iterative approximation of the normal RPE position in drusen described above, a smooth function is computed to closely fit to its posterior border. This resulting smooth function is used as the spine of the evaluation band, which extends $15 \Delta z_{\text {pix }}$ in the anterior and posterior directions. This width ensures that depolarizing spots in the choroid are excluded from the band, yet allowing for small irregularities of the axial RPE position such as small drusen because they are being found in dry AMD.

By summing the number of depolarizing pixels within the evaluation band along each A-line, an RPE thickness map is generated. In order to assess the size of atrophic zones, the thickness map is binarized and smoothed by removing isolated 

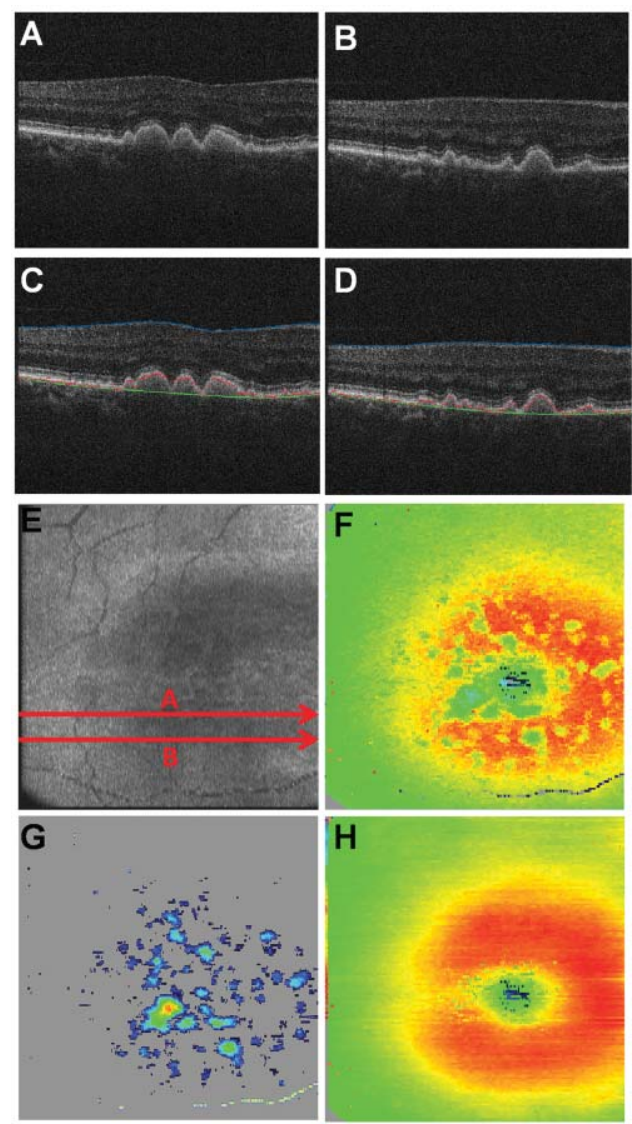

Fig. 3 Results from drusen segmentation. (a, b) Reflectivity B-scan images. (c, d) Overlaid positions of ILM (blue), RPE (red), and normal RPE (green). (e) En face fundus image reconstructed from 3-D OCT data set. (f) Retinal thickness map showing the axial distance between ILM and RPE position (color map: 70-350 $\mu \mathrm{m}$ ). (g) Drusen thickness map computed as axial distance between segmented RPE position and approximated normal RPE position. The color map was scaled to the maximum elevation value of 55 pixels corresponding to $\sim 128 \mu \mathrm{m}$. (h) Retinal thickness map showing the axial distance between ILM and computed normal RPE position (color map: 70-350 $\mu \mathrm{m}$ ).

pixels using an erosion filter. In a last step, the algorithm automatically detects patches of connected pixels, which can now be converted into atrophic areas by scaling them with the known pixel area.

\section{Results}

\subsection{Drusen}

Figure 3 shows results from drusen segmentation in a PS-OCT data set recorded in the right eye of a female patient at age 70 . Reflectivity B-scan images are shown in Figs. 3(a) and 3(b). In Figs. 3(c) and 3(d), an overlay of the segmented ILM and RPE as well as of the computed normal RPE position is displayed in blue, red, and green color, respectively. The fundus projection image is shown in Fig. 3(e); the positions of the B-scans shown in Figs. 3(a)-3(d) are indicated by red arrows. A retinal thickness map computed as the axial distance between ILM and segmented actual RPE position is shown in Fig. 3(f), while for the thickness map in Fig. 3(h), the axial distance between ILM and the approximated normal RPE position were used. In these maps, single pixels exhibiting thickness values of $>150$ pixels (corresponding to a retinal thickness of $350 \mu \mathrm{m}$ ) were linearly interpolated between neighboring pixels with values of $<350 \mu \mathrm{m}$. Finally, a drusen thickness map displaying the axial deviation of the actual RPE position from the computed normal RPE position is shown in Fig. 3(g). Deviation values of $<8$ pixels $(25.6 \mu \mathrm{m})$ were tolerated as small variations

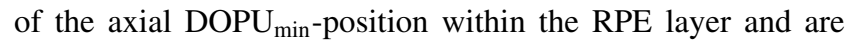
displayed gray.

From the drusen thickness map [Fig. 3(g)], drusen dimensions can be assessed quantitatively. Namely, their area and volume can be computed from the known area of a pixel in the en face image $\left[\sim 12.5(x) \times 50.2(y) \approx 630 \mu \mathrm{m}^{2}\right]$ and the axial extension of one voxel ( $\sim 3.2 \mu \mathrm{m}$, optical distance). For the data set shown in Fig. 3, the total area of drusen in the scanned region of the retina was calculated to be $5.527 \mathrm{~mm}^{2}$ with a volume of $0.188 \mathrm{~mm}^{3}$. An average refractive index of 1.38 was assumed for drusen volume computation.

\subsection{Geographic Atrophy}

Results from applying the segmentation algorithms to the right eye of an 80-year-old female patient diagnosed with dry AMD are shown in Fig. 4. In Fig. 4(a), an en face fundus image computed from the 3-D data set is shown. The red arrows indicate

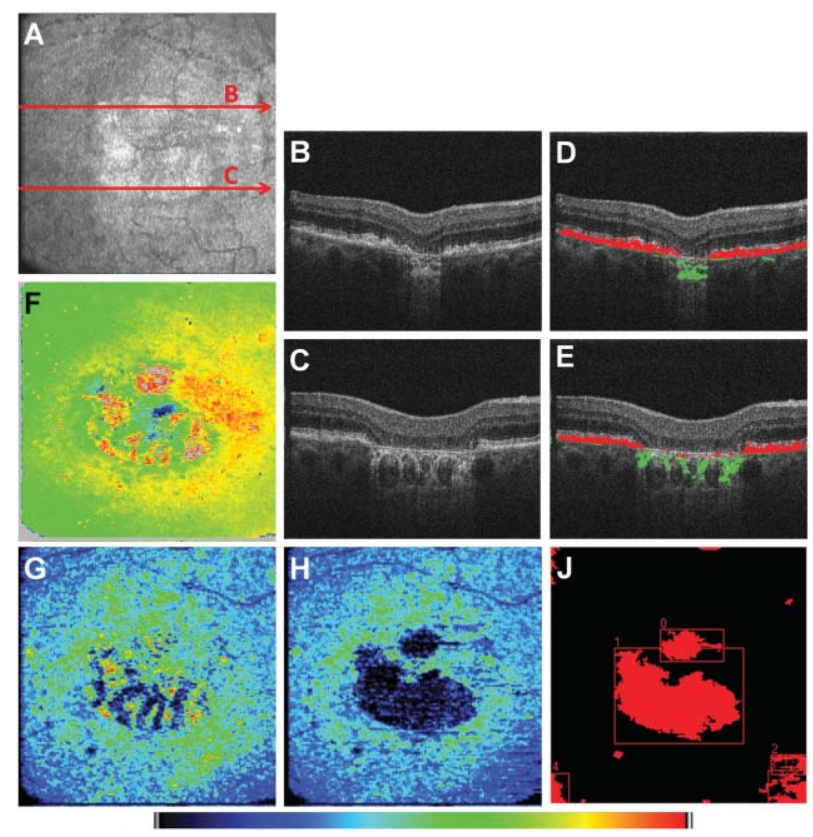

Fig. 4 Geographic atrophy: (a) En face fundus image reconstructed from 3-D OCT data set, (b, c) reflectivity B-scan images, $(d$, e) Overlay of depolarizing structures within and outside the evaluation band shown in red and green color, respectively, and ( $f$ ) retinal thickness map computed as axial distance between ILM and DOPU $\min$ position. The DOPU $_{\text {min }}$ position is usually located in the RPE. In the case of atrophy, this position was located in the choroid [green pixels in (d) and (e)]. (g) Map of overall number of depolarizing pixels per A-line. Zones of RPE atrophy are masked by choroidal depolarization. (h) Map displaying the thickness of the depolarizing layer inside the evaluation band. (j) Binary map of atrophic zones. The color map scales from 70 to $325 \mu \mathrm{m}$ for (f) and from 0 to 39 pixels for $(\mathrm{g})$ and $(\mathrm{h})$. 
the position of the reflectivity B-scans of Figs. 4(b) and 4(c). In Figs. 4(d) and 4(e), an overlay of depolarizing structures inside and outside of the evaluation band is shown in red and green, respectively. The retinal thickness computed as axial distance between ILM and DOPU $\mathrm{Um}_{\min }$ position is mapped in Fig. 4(f). In the map shown Fig. 4(g), the overall thickness of depolarizing tissue [i.e., red and green pixels in Figs. 4(d) and 4(e)] is displayed, while for the thickness map of Fig. 4(h), only depolarizing pixels within the evaluation band (i.e., the red pixels in the B-scan images) were considered as the depolarizing layer. After processing this image as described in Section 2.4, two atrophic zones, sized 0.570 and $3.841 \mathrm{~mm}^{2}$, were found automatically. Zones that were connected to the image border [cf. lower left and right corner of Fig. 4(j)] were excluded by the algorithm from the calculation of the total area. The total area of geographic atrophy was $4.412 \mathrm{~mm}^{2}$ for this data set.

\subsection{Repeatability of Assessed Pathology Dimensions}

In order to check the repeatability of the areas and volumes determined for drusen and atrophic zones, respectively, repeated measurements during a single visit were performed in several patients. Five data sets each, from seven eyes diagnosed with drusen and from five eyes diagnosed with geographic atrophy were evaluated. For both the drusen and geographic atrophy data sets, a fixed value of DOPU $U_{\text {thr }}=0.8$ was chosen for RPE segmentation. For every eye diagnosed with drusen, the drusen thickness maps were registered and cropped to the same retinal section using the OCT fundus image and Image J. Then, the area and volume of drusen were computed and analyzed. In the case of geographic atrophy, only such atrophic zones were integrated into area calculation that did not exceed the OCT device's scan area in all of the five maps. For that reason, one atrophic zone was excluded in one of the investigated eyes.

Results from repeatability measurements can be found in Tables 1 and 2 as well as in Figs. 5 and 6 for drusen, and in Table 3 and Fig. 7 for the evaluation of geographic atrophy segmentation. Drusen areas in the range of $3.225-9.802 \mathrm{~mm}^{2}$

Table 1 Repeatability of drusen area measurements.

\begin{tabular}{cccc}
\hline Eye ID & Average area $^{*}$ & $s^{*}$ & Coefficient of variation \\
\hline 1 & 3.225 & 0.405 & 0.126 \\
2 & 3.844 & 0.240 & 0.063 \\
3 & 4.120 & 0.434 & 0.105 \\
4 & 8.251 & 0.493 & 0.060 \\
5 & 9.802 & 0.721 & 0.074 \\
6 & 5.697 & 0.473 & 0.083 \\
7 & 5.207 & 0.169 & 0.032 \\
Average & 5.735 & 0.419 & 0.077 \\
\hline
\end{tabular}

*Average area and standard deviation of determined drusen areas are given in millimeters squared. Average values of all eyes are given in the last line.
Table 2 Repeatability of drusen volume measurements.

\begin{tabular}{cccc}
\hline Eye ID & Average volume $^{*}$ & $\mathrm{sd}^{*}$ & Coefficient of variation $^{*}$ \\
\hline 1 & 0.084 & 0.007 & 0.080 \\
2 & 0.095 & 0.009 & 0.100 \\
3 & 0.133 & 0.010 & 0.076 \\
4 & 0.456 & 0.018 & 0.040 \\
5 & 0.362 & 0.023 & 0.062 \\
6 & 0.168 & 0.018 & 0.107 \\
7 & 0.179 & 0.010 & 0.057 \\
Average & 0.211 & 0.014 & 0.075 \\
\hline
\end{tabular}

*Average volume and standard deviation of determined drusen volumes are given in cubic millimeters. Average values of all eyes are given in the last line.

were measured. The average area was $5.735 \mathrm{~mm}^{2}$. Measured areas varied by $0.169-0.721 \mathrm{~mm}^{2}$ [standard deviation (sd)] with a mean variation of $0.419 \mathrm{~mm}^{2}$. The coefficient of variation $(\mathrm{sd} / \mathrm{mean})$ was in average 0.077 , ranging from 0.032 to 0.126 . For the same eyes, drusen volumes ranging from 0.084 to $0.456 \mathrm{~mm}^{3}\left(\right.$ mean value $=0.211 \mathrm{~mm}^{3} ;$ mean $\left.\mathrm{sd}=0.014 \mathrm{~mm}^{3}\right)$ were observed. Variations between 0.007 and $0.023 \mathrm{~mm}^{3}$ (sd) were computed. The average coefficient of variation was determined to be 0.075 (range: $0.040-0.107$ ).

Segmentation of geographic atrophy yielded areas between 0.620 and $7.788 \mathrm{~mm}^{2}$ (mean value $=3.132 \mathrm{~mm}^{2}$, mean sd $=0.326 \mathrm{~mm}^{2}$ ). The variation of determined areas resulted in standard deviations in the range of $0.056-0.979 \mathrm{~mm}^{2}$ and in coefficients of variation between 0.036 and 0.140 , with an average of 0.092 .

The box plots shown in Figs. 5-7 illustrate the distribution of measured values. The upper and lower edges of the box indicate the 25 and $75 \%$ quartile, whereas the whiskers show the minimum and maximum of the measured areas and volumes, respectively. The bar inside the box represents the median.

\section{Discussion}

In the results shown in Section 3, the performance of the introduced segmentation algorithms may be appreciated. Although the method seems to perform well in the eyes shown in Figs. 3 and 4 , the repeatability measurements show variability values of 7.7 and $7.5 \%$ for determined drusen areas and volumes, and a coefficient of variation of $9.2 \%$ for areas of geographic atrophy. These variations correspond to $1 \%$ (661 pixels) and $0.8 \%$ (514 pixels) of the total scanned area for drusen area and geographic atrophy segmentation, respectively. In the case of drusen volume assessment, the COV of $7.5 \%$ corresponds to $0.01 \%(6702$ voxels) of the total scanned volume. We believe that these variations are mainly due to eye motion during the measurement. For the PS-OCT instrument used for this study, it takes roughly $3.3 \mathrm{~s}$ to record one 3-D data set. Any fixation instability and (transverse) eye motion during that period will inevitably result in distortion of the reconstructed en face images, such as 


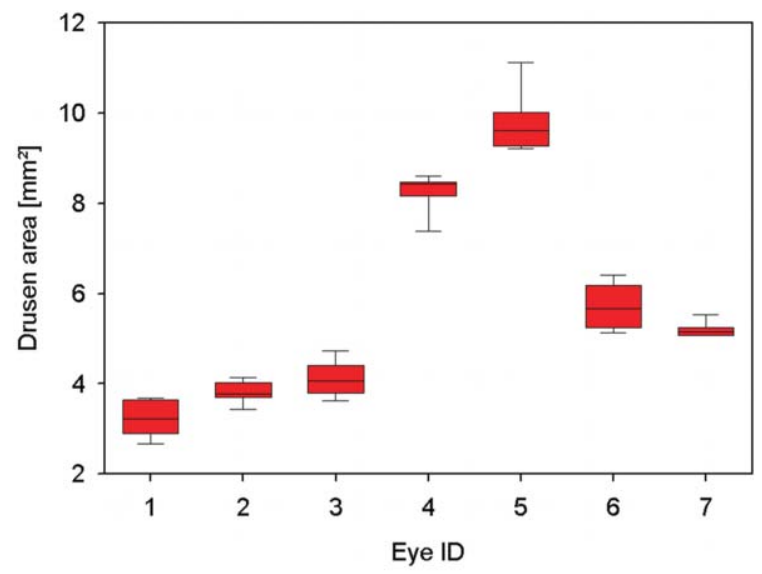

(a)

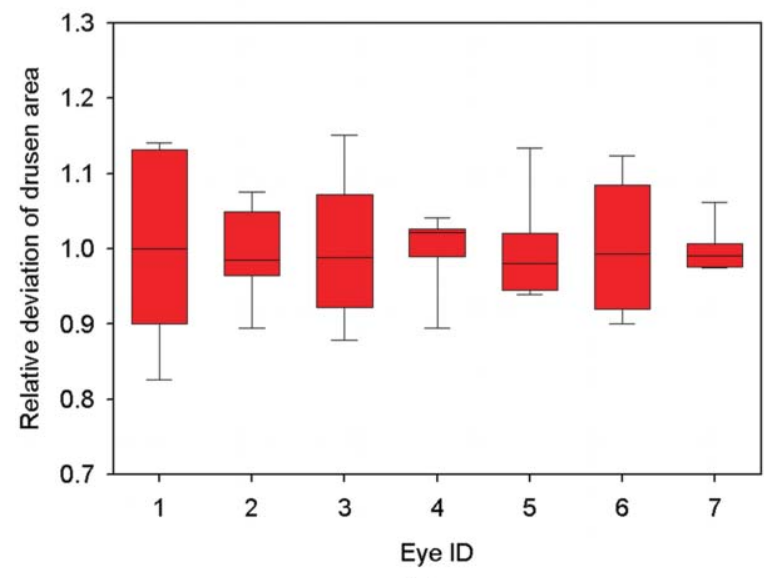

(b)

Fig. 5 Repeatability of drusen area measurements. (a) For seven eyes, box plots of drusen area measurements from five repeated measurements are shown. (b) Box plots of relative deviation of the areas for the same data sets.

OCT fundus images, drusen thickness maps, or thickness maps of depolarizing tissue. Hence, any quantitative data, such as areas or volumes retrieved from these maps, will be affected. In order to increase repeatability even more, either transverse eye motion has to be compensated for or acquisition speed has to be increased. Transverse motion compensation (especially along the slow scanning $Y$ direction) may be achieved for instance by registering the recorded 3-D OCT data to SLO images from the same fundus region during postprocessing, because SLO images can be recorded much faster than 3-D OCT data sets. A hardware solution for transverse motion compensation would be using a retinal tracker for stabilizing the probe beam with respect to the retina under investigation. ${ }^{37}$ Recent developments in light source and data-acquisition technology have enabled line rates of several 100,000 A-lines per second, which is more than 10 times the line rate of the PS-OCT instrument we used, which can further reduce motion artifacts. ${ }^{38,39}$ However, even the variation of geographic atrophy area determined from our repeatability measurements [i.e., $\sim 0.33 \mathrm{~mm}^{2}$ (cf. Table 3)] is still significantly smaller than the area growth rate of $1.52 \mathrm{~mm}^{2} / \mathrm{yr}$ and $4.57 \mathrm{~mm}^{2} / 2 \mathrm{yr}$ determined by using FAF and fundus photography reported in literature..$^{40,41}$

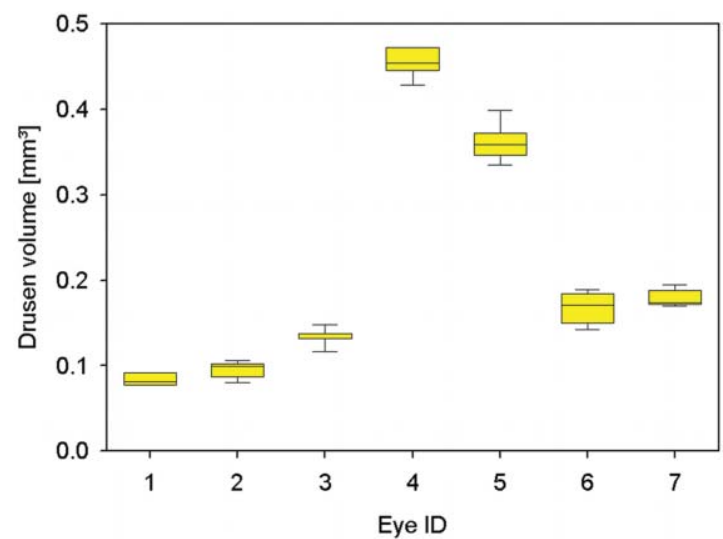

(a)

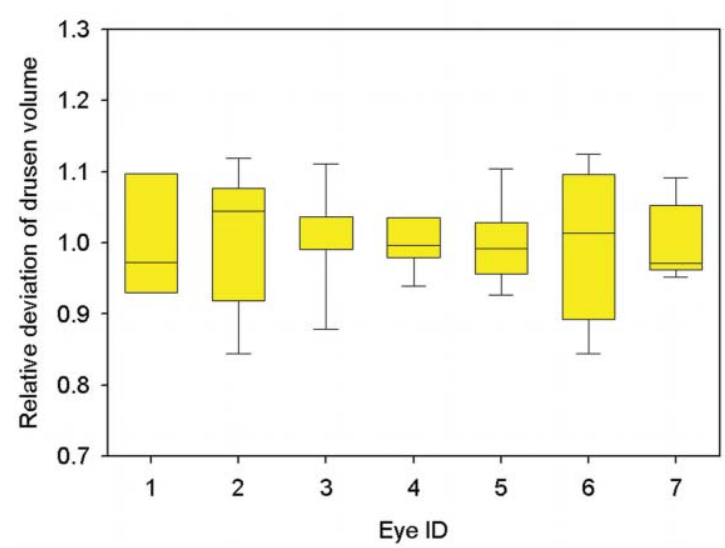

(b)

Fig. 6 Repeatability of drusen volume measurements. (a) For seven eyes, box plots of drusen volume measurements from five repeated measurements are shown. (b) Box plots of relative deviation of the volumes for the same data sets.

One advantage of PS-OCT-based segmentation as compared to-clinically eshablished-imaging modalities, such as fundus photography, FAF, or scanning laser polarimetry, is its ability for depth-resolved imaging. Although all of the aforementioned techniques have proven to be able to image and discriminate pigmented structures, the images will only be the results of a depth integration. Therefore, signals from structures lying on each

Table 3 Repeatability of geographic atrophy area measurements.

\begin{tabular}{cccc}
\hline Eye ID & Average area $^{*}$ & $\mathrm{sd}^{*}$ & Coefficient of variation \\
\hline 1 & 2.215 & 0.157 & 0.071 \\
2 & 2.470 & 0.345 & 0.140 \\
3 & 0.620 & 0.056 & 0.090 \\
4 & 7.788 & 0.979 & 0.126 \\
5 & 2.564 & 0.092 & 0.036 \\
Average & 3.132 & 0.326 & 0.092 \\
\hline
\end{tabular}

*Average area and standard deviation of determined areas are given in millimeters squared. Average values of all eyes are given in the last line. 


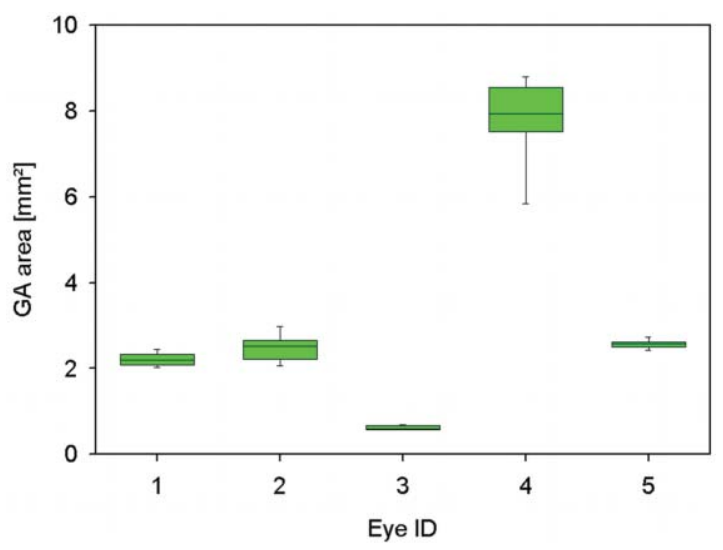

(a)

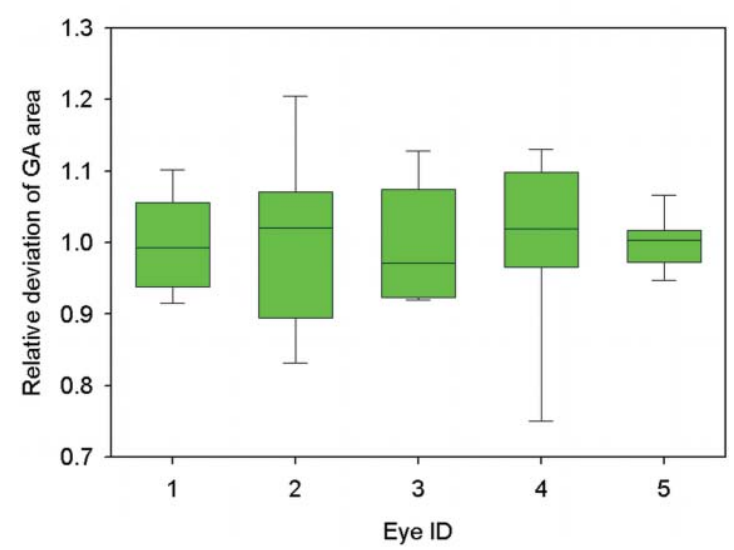

(b)

Fig. 7 Repeatability of area measurements in geographic atrophy. (a) For five eyes, box plots of geographic atrophy area measurements from five repeated measurements are shown. (b) Box plots of relative deviation of the areas for the same data sets.

other will add in the resulting image, which can lead to masking or mimicking of pathology as well as to misinterpretation of retinal features. In contrast, PS-OCT enables discrimination and localization of pigmented structures like the RPE in three dimensions.

It also must be pointed out that, although the area and volume data presented in this paper showed the overall pathology areas and volumes in the respective 3-D data set, the drusen and geographic atrophy maps could also be used to assess the dimensions of single features such as individual druses or atrophic patches.

The results presented in this study were based on twodimensionally operating segmentation algorithms; that is, segmentation of the RPE was performed B-scan by B-scan. Evaluation in three dimensions could even improve segmentation results because they would enable one to apply smaller evaluation kernels for DOPU computation as well as probably even smoother area and volume assessment in three dimensions. Such further improvements of the segmentation algorithms might also increase data-evaluation speed. Currently, using a stateof-the-art personal computer (Intel Core 2 Quad processor, $4 \times 2.50 \mathrm{GHz}, 4 \mathrm{~GB}$ RAM, Windows XP) data evaluation from raw data to saving PS-OCT B-scan images and maps takes $\sim 1.4$ and $\sim 2.3$ s per B-scan for segmenting and evaluating drusen and geographic atrophy, respectively (including loading data from a USB hard drive).

\section{Conclusion}

In this paper, we presented new applications of PS-OCT for segmentation and quantitative assessment of retinal pathologies in AMD. Area and volume of macular drusen were determined as well as areas of atrophic zones in late stage dry AMD. The segmentation algorithms were based on the polarization scrambling characteristics of the RPE, which allows distinguishing this layer from other retinal layers. The assessed pathology dimensions showed good repeatability and, once again, presented PS-OCT as a versatile method for ophthalmic imaging. PS-OCT might be a promising technique for imaging of retinal pathology in everyday ophthalmology.

\section{Acknowledgments}

The authors thank Martin Wurm and David Stifter at RECENDT $\mathrm{GmbH}$ as well as Christoph Wölfl at the Medical University of Vienna for their excellent technical support. Financial support by the Austrian Science Fund (FWF Grant No. P19624-B02) and from the European Union project FUN OCT (FP7 HEALTH, Contract No. 201880) is gratefully acknowledged.

\section{References}

1. D. Huang, E. A. Swanson, C. P. Lin, J. S. Schuman, W. G. Stinson, W. Chang, M. R. Hee, T. Flotte, K. Gregory, C. A. Puliafito, and J. G. Fujimoto, "Optical coherence tomography," Science 254, 1178-1181 (1991).

2. W. Drexler and J. G. Fujimoto, Optical Coherence TomographyTechnology and Applications, Springer, Berlin (2008).

3. A. F. Fercher, W. Drexler, C. K. Hitzenberger, and T. Lasser, "Optical coherence tomography_-principles and applications," Rep. Prog. Phys. 66, 239-303 (2003)

4. W. Drexler and J. G. Fujimoto, "State-of-the-art retinal optical coherence tomography," Prog. Ret. Eye Res. 27, 45-88 (2008).

5. R. A. Leitgeb, C. K. Hitzenberger, and A. F. Fercher, "Performance of Fourier domain vs. time domain optical coherence tomography," Opt. Express 11, 889-894 (2003).

6. J. F. de Boer, B. Cense, B. H. Park, M. C. Pierce, G. J. Tearney, and B. E. Bouma, "Improved signal to noise ratio in spectral domain compared with time domain optical coherence tomography," Opt. Lett. 28, 2067-2069 (2003).

7. M. A. Choma, M. V. Sarunic, C. Yang, and J. A. Izatt, "Sensitivity advantage of swept source and Fourier domain optical coherence tomography," Opt. Express 11, 2183-2189 (2003).

8. D. Koozekanani, K. Boyer, and C. Roberts, "Retinal thickness measurements from optical coherence tomography using a Markov boundary model," IEEE Trans. Med. Imaging 20, 900-916 (2001).

9. H. Ishikawa, D.M. Stein, G. Wollstein, S. Beaton, J. G. Fujimoto, and J. S. Schuman, "Macular segmentation with optical coherence tomography," Invest. Ophthalmol. Vis. Sci. 46, 2012-2017 (2005).

10. M. Mujat, R. C. Chan, B. Cense, B. H. Park, C. Joo, T. Akkin, T. C. Chen, and J. F. de Boer, "Retinal nerve fiber layer thickness map determined from optical coherence tomography images," Opt. Express 13, 9480-9491 (2005).

11. M. Szkulmowski, M. Wojtkowski, B. Sikorski, T. Bajraszewski, V. J. Srinivasan, A. Szkulmowska, J. J. Kaluzny, J. G. Fujimoto, and A. Kowalczyk, "Analysis of posterior retinal layers in spectral optical coherence tomography images of the normal retina and retinal pathologies," J. Biomed. Opt. 12, 041207 (2007).

12. D. C. Fernandez, H. M. Salinas, and C. A. Puliafito, "Automated detection of retinal layer structures on optical coherence tomography images," Opt. Express 13, 10200-10216 (2005). 
13. M. Baroni, P. Fortunato, and A. L. Torre, "Towards quantitative analysis of retinal features in optical coherence tomography," Med. Eng. Phys. 29, 432-441 (2007).

14. T. Fabritius, S. Makita, M. Miura, R. Myllylä, and Y. Yasuno, "Automated segmentation of the macula by optical coherence tomography," Opt. Express 17, 15659-15669 (2009).

15. A. Mishra, A. Wong, K. Bizheva, and D. A. Clausi, "Intra-retinal layer segmentation in optical coherence tomography images," Opt. Express 17, 23719-23728 (2009).

16. X. J. Wang, T. E. Milner, and J. S. Nelson, "Characterization of fluid flow velocity by optical Doppler tomography," Opt. Lett. 20, 1337-1339 (1995).

17. Z. Chen, T. E. Milner, D. Dave, and J. S. Nelson, "Optical Doppler tomographic imaging of fluid flow velocity in highly scattering media," Opt. Lett. 22, 64-66 (1997)

18. U. Morgner, W. Drexler, F. X. Kärtner, X. D. Li, C. Pitris, E. P. Ippen, and J. G. Fujimoto, "Spectroscopic optical coherence tomography," Opt. Lett. 25, 111-113 (2000).

19. M. R. Hee, D. Huang, E. A. Swanson, and J. G. Fujimoto, "Polarization sensitive low coherence reflectometer for birefringence characterization and ranging," J. Opt. Soc. Am. B 9, 903-908 (1992).

20. J. F. de Boer, T. E. Milner, M. J. C. van Gemert, and J. S. Nelson, "Twodimensional birefringence imaging in biological tissue by polarizationsensitive optical coherence tomography," Opt. Lett. 22, 934-936 (1997).

21. B. Cense, T. C. Chen, B. Hyle Park, M. C. Pierce, and J. F. de Boer, "Thickness and birefringence of healthy retinal nerve fiber layer tissue measured with polarization-sensitive optical coherence tomography," Invest. Ophthalmol. Vis. Sci. 45, 2606-2612 (2004).

22. M. Pircher, E. Götzinger, R. Leitgeb, H. Sattmann, O. Findl, and C. K. Hitzenberger "Imaging of polarization properties of human retina in vivo with phase resolved transversal PS-OCT," Opt. Express 12 5940-5951 (2004).

23. M. Pircher, E. Götzinger, O. Findl, S. Michels, W. Geitzenauer, C. Leydolt, U. Schmidt-Erfurth, and C. K. Hitzenberger, "Human macula investigated in vivo with polarization sensitive optical coherence tomography," Invest. Ophthalmol. Vis. Sci. 47, 5487-5494 (2006).

24. M. Yamanari, M. Miura, S. Makita, T. Yatagai, and Y. Yasuno, "Phase retardation measurement of retinal nerve fiber layer by polarizationsensitive spectral-domain optical coherence tomography and scanning laser polarimetry," J. Biomed Opt. 13, 014013 (2008).

25. E. Götzinger, M. Pircher, B. Baumann, C. Ahlers, W. Geitzenauer, U. Schmidt-Erfurth, and C. K. Hitzenberger, "Three-dimensional polarization sensitive OCT imaging and interactive display of the human retina," Opt. Express 17, 4151-4165 (2009).

26. E. Götzinger, M. Pircher, W. Geitzenauer, C. Ahlers, B. Baumann, S. Michels, U. Schmidt-Erfurth, and C. K. Hitzenberger, "Retinal pigment epithelium segmentation by polarization sensitive optical coherence tomography," Opt. Express 16, 16410-16422 (2008).

27. C. Ahlers, E. Goetzinger, M. Pircher, I. Golbaz, F. Prager, C. Schütze, B. Baumann, C. Hitzenberger, and U. Schmidt-Erfurth, "Imaging of the retinal pigment epithelium in age-related macular degeneration using polarization sensitive optical coherence tomography," Invest. Ophthalmol. Vis. Sci. 51, 2149-2157 (2009).
28. S. Michels, M. Pircher, W. Geitzenauer, C. Simader, E. Götzinger, O. Findl, U. Schmidt-Erfurth, and C. K. Hitzenberger, "Value of polarisation-sensitive optical coherence tomography in diseases affecting the retinal pigment epithelium,” Br. J. Ophthalmol. 92, 204-209 (2008).

29. R. Klein, B. E. Klein, and K. L. Linton, "Prevalence of age-related maculopathy: the Beaver Dam Eye Study," Ophthalmology 99, 933943 (1992).

30. A. C. Bird, N. M. Bressler, S. B. Bressler, I. H. Chisholm, G. Coscas, M. D. Davis, P. T. V. M. de Jong, C. C. W. Klaver, B. E. K. Klein, R. Klein, P. Mitchell, J. P. Sarks, S. H. Sarks, G. Soubrane, H. R. Taylor, and J. R. Vingerling, "An international classification and grading system for age-related maculopathy and age-related macular degeneration," Surv. Ophthalmol. 39, 367-374 (1995).

31. U. M. Schmidt-Erfurth and C. Pruente, "Management of neovascular agerelated macular degeneration," Prog. Ret. Eye Res. 26, 437-451 (2007).

32. LIA, "American national standard for safe use of lasers," ANSI Z 136.1, Orlando (2000).

33. InternationaI Electrotechnical Comission, "Safety of laser productsPart 1: equipment classication and requirements," IEC 60825-1 Ed. 2 (2001).

34. C. K. Hitzenberger, E. Götzinger, M. Sticker, M. Pircher, and A. F. Fercher, "Measurement and imaging of birefringence and optic axis orientation by phase resolved polarization sensitive optical coherence tomography," Opt. Express 9, 780-790 (2001).

35. M. Wojtkowski, V. Srinivasan, T. Ko, J. Fujimoto, A. Kowalczyk, and J. Duker, "Ultrahigh-resolution, high-speed, Fourier domain optical coherence tomography and methods for dispersion compensation," Opt. Express 12, 2404-2422 (2004)

36. B. Baumann, E. Götzinger, M. Pircher, and C. K. Hitzenberger, "Measurement of depolarization distribution in the healthy human macula by polarization sensitive OCT," J. Biophoton. 2, 426-434 (2009).

37. R. D. Ferguson, D. X. Hammer, L. A. Paunescu, S. Beaton, and J. S. Schuman, "Tracking optical coherence tomography," Opt. Lett. 29, 2139-2141 (2004).

38. R. Huber, M. Wojtkowski, and J. G. Fujimoto, "Fourier domain mode locking (FDML): a new laser operating regime and applications for optical coherence tomography," Opt. Express 14, 3225-3237 (2006).

39. B. Potsaid, I. Gorczynska, V. J. Srinivasan, Y. Chen, J. Jiang, A. Cable, and J. G. Fujimoto, "Ultrahigh speed spectral/Fourier domain OCT ophthalmic imaging at 70,000 to 312,500 axial scans per second," Opt. Express 16, 15149-15169 (2008).

40. F. G. Holz, A. Bindewald-Wittich, M. Fleckenstein, J. Dreyhaupt, H. P. N. Scholl, S. Schmitz-Valckenberg, and Fam-Study Group, "Progression of geographic atrophy and impact of fundus autofluorescence patterns in age-related macular degeneration," Am. J. Ophthalmol. 143, 463-472 (2007)

41. J. S. Sunness, J. Gonzalez-Baron, C. A. Applegate, N. M. Bressler, Y. Tian, B. Hawkins, Y. Barron, and A. Bergman, "Enlargement of atrophy and visual acuity loss in the geographic atrophy form of age-related macular degeneration," Ophthalmology 106, 1768-1779 (1999). 